\title{
Securely Computing an Approximate Median in Wireless Sensor Networks.
}

\section{Sankardas Roy ${ }^{1}$ sroy1@gmu.edu}

\author{
Mauro Conti $i^{1,2}$ \\ conti@di.uniroma1.it
}

\author{
Sanjeev Setia ${ }^{3}$ \\ setia@gmu.edu
}

\author{
Sushil Jajodia ${ }^{1}$ \\ jajodia@gmu.edu
}

${ }^{1}$ Center for Secure Information Systems, George Mason University, Fairfax, VA, USA - 22030

2 Dipartimento di Informatica, Università di Roma "La Sapienza", 00198 Roma, Italy

${ }^{3}$ Department of Computer Science, George Mason University, Fairfax, VA, USA - 22030

\begin{abstract}
Wireless Sensor Networks (WSNs) have proven to be useful in many applications, such as military surveillance and environment monitoring. To meet the severe energy constraints in WSNs, some researchers have proposed to use the in-network data aggregation technique (i.e., combining partial results at intermediate nodes during message routing), which significantly reduces the communication overhead. Given the lack of hardware support for tamper resistance and the unattended nature of sensor nodes, sensor network protocols need to be designed with security in mind. Recently, researchers proposed algorithms for securely computing a few aggregates, such as Sum (the sum of the sensed values), Count (number of nodes) and Average. However, to the best of our knowledge, there is no prior work which securely computes the Median, although the Median is considered to be an important aggregate. The contribution of this paper is twofold. We first propose a protocol to compute an approximate Median and verify if it has been falsified by an adversary. Then, we design an attack-resilient algorithm to compute the Median even in the presence of a few compromised nodes. We evaluate the performance and cost of our approach via both analysis and simulation. Our results show that our approach is scalable and efficient.
\end{abstract}

\section{Categories and Subject Descriptors}

C.2.0 [Computer-Communication Networks]: GeneralSecurity and protection; C.2.1 [Computer-Communication Networks]: Network Architecture and Design-Wireless communication; D.4.6 [Operating Systems]: Security and Protection-Cryptographic controls; K.6.5 [Management of Computing and Information Systems]: Security and Protection

*The work of Sushil Jajodia is partially supported by National Science Foundation under grants CT-0716567, CT0627493, IIS-0242237, and IIS-0430402.

Permission to make digital or hard copies of all or part of this work for personal or classroom use is granted without fee provided that copies are not made or distributed for profit or commercial advantage and that copies bear this notice and the full citation on the first page. To copy otherwise, to republish, to post on servers or to redistribute to lists, requires prior specific permission and/or a fee.

SecureComm 2008 September 22-25, 2008, Istanbul,Turkey

Copyright 2008 ACM 978-1-60558-241-2 ...\$5.00.

\section{General Terms}

Algorithms, Design, Security

\section{Keywords}

Sensor Network Security, Data Aggregation, Hierarchical Aggregation, Attack-Resilient

\section{INTRODUCTION}

Wireless Sensor Networks (WSNs) are being used in many applications [13, 11, 24], such as military surveillance, wildlife habitat monitoring, forest fire prevention, etc. A WSN normally consists of a large number of sensor nodes which are self-organized into a multi-hop network.

The simplest way to collect the sensed data is to let each sensor node deliver its reading to the base station (BS). This approach, however, is wasteful since it results in excessive communication. A typical sensor node is severely constrained in communication bandwidth and energy reserve. Hence, sensor network designers have advocated alternative approaches for data collection.

An in-network aggregation algorithm combines partial results at intermediate nodes during message routing, which significantly reduces the amount of communication and hence the energy consumed. A typical data acquisition system [14, $8]$ constructs a spanning tree rooted at the BS and then performs in-network aggregation along the tree. Partial results propagate level by level up the tree, with each node awaiting messages from all of its children before sending a new partial result to its parent. Researchers $[14,8]$ have designed several energy-efficient algorithms to compute aggregates such as Count, Sum, Average, etc. However, an in-network aggregation algorithm cannot cheaply compute the exact Median, where the worst case communication overhead per node is $\Omega(N)$, where $N$ is the number of nodes in the network [14]. As a result, researchers have advocated computation of an approximate Median. In-network aggregation algorithms to compute an approximate Median are proposed in [10, 23].

Unfortunately, none of the above algorithms include any provisions for security, and hence, they cannot be used in security-sensitive applications. Given the lack of tamperresistance and the unattended nature of many networks, we must consider the possibility that a few sensor nodes in the network might become compromised.

A compromised node in the aggregation hierarchy may attempt to change the aggregate value computed at the BS by relaying a false sub-aggregate value to its parent. This at- 


\begin{tabular}{|c|c|c|c|c|}
\hline & $\begin{array}{c}\text { Node } \\
\text { congestion }\end{array}$ & $\begin{array}{c}\text { Latency } \\
\text { (epochs) }\end{array}$ & Verification & $\begin{array}{c}\text { Attack-resilient } \\
\text { computation }\end{array}$ \\
\hline \hline Greenwald et al.'s protocol [10] & $O\left(\left(\log ^{2} N\right) / \epsilon\right)$ & 2 & No & No \\
\hline GC approach (Section 4.1) & $O\left(\left(\log ^{2} N\right) / \epsilon\right)$ & 6 & Yes & No \\
\hline Our basic protocol (Section 4.3) & $O((1 / \epsilon) \Delta \log N)$ & 6 w.h.p. & Yes & No \\
\hline Our extended protocol (Section 6) & $O((1 / \epsilon) \Delta \log N)$ & 6 w.h.p. & Yes & Yes \\
\hline
\end{tabular}

Table 1: Median computation protocols: Comparing the performance and the security features

tack can be launched on most of the in-network aggregation algorithms. For example, in Greenwald et al.'s approximate Median computation algorithm [10], a compromised node in the aggregation hierarchy can corrupt the quantile summary to make the BS accept a false Median which might contain a large amount of error.

A technique to compute and verify Sum and Count aggregates has been recently proposed by Chan et al. [3]. Their scheme [3] can also verify if a given value is the true Median, but they have not proposed any solution to compute that value in the first place. To the best of our knowledge, there is no prior work which securely computes the Median using an in-network algorithm.

One might suggest an approach which runs Greenwald et al.'s algorithm [10] to compute an approximate Median and then employs Chan et al.'s verification protocol [3] to verify if the computed value is indeed a valid estimate. We refer this approach as GC in the rest of the paper. The communication cost per node in this approach is $O\left(\frac{\log ^{2} N}{\epsilon}\right)$, where $\epsilon$ is the approximation error bound.

In this paper, we propose an alternative approach to compute and verify an approximate Median, which proves to be more efficient compared to the GC approach. Our approach is based on sampling - an uniform sample of sensed values is collected from the network to make a preliminary estimate of the Median, which is verified and refined later. The communication cost of our basic algorithm is $O\left(\frac{1}{\epsilon} \Delta \log N\right)$, where $\epsilon$ is the error bound and $\Delta$ is the maximum degree of the aggregation tree used by the algorithm.

Like the GC approach, our basic algorithm guarantees that an attacker cannot cause the BS to accept a Median estimate which contains an error more than the user-specified bound, $\epsilon$. However, neither of the above approaches can guarantee the successful computation of the Median in the presence of an attacker. To address this problem, we extend the basic approach so that we can compute the Median even in the presence of a few compromised nodes. The analysis and simulation results show that our algorithms are effective and efficient. Further, our algorithms can be extended to compute other quantiles.

Table 1 compares our approach with other solutions on the basis of a few performance and security metrics. We report node congestion as a metric for communication complexity, which represents the worst case overhead on a single node. We measure the latency of the protocols in epochs. Similarly to the prior work [14], an epoch represents the amount of time a message takes to traverse the distance between the $\mathrm{BS}$ and the farthest node on the aggregation hierarchy. We observe that the latency of our protocol might increase in extreme cases; here we report the latency which our protocol incurs in most cases (i.e., with high probability (w.h.p.)). To measure the security of the protocols, we consider the following properties. We say that a protocol has verification property if the protocol enables the BS to verify whether the computed Median is false or not. Observe that this property does not guarantee the computation of the Median in the presence of an attack. Finally, an attack-resilient protocol is so if it guarantees the computation of the Median in the presence of a few malicious nodes.

Organization The rest of the paper is organized as follows. In Section 2, we review the related work present in the literature. Section 3 describes the problem and the assumptions taken in this paper. In Section 4, we present our basic protocol, whose security and performance analysis is given in Section 5. Section 6 describes our attack-resilient protocol. We present our simulation results in Section 7, and finally, we conclude the paper in Section 8.

\section{RELATED WORK}

Several researchers $[14,8]$ have proposed in-network aggregation algorithms which fuse the sensed information en route to the $\mathrm{BS}$ to reduce the communication overhead. In particular, these algorithms are designed to compute algebraic aggregates, such as Sum, Count, and Average. However, Madden et al. [14] showed that in-network aggregation does not save any communication overhead in case of computing holistic aggregates, such as the Median.

To limit the communication complexity, researchers have advocated computing an approximate estimate instead of the exact Median [10, 23]. In particular, Greenwald et al. [10] proposed a quantile summary computation algorithm that exploits a concept of delayed aggregation so that no summary contains error more than $\epsilon$ bound. Also, Srivastava et al. [23] presented another data summarization technique called quantile digest to compute an approximate median, where the main idea is to compute an equi-depth histogram through in-network aggregation. There also exists a body of data stream algorithms in the literature which computes approximate quantiles $[9,15,5]$. In fact, Greenwald et al.'s algorithm [10] is an extension of [9].

Our Median computation algorithm has a sampling phase and a histogram computation phase. Sampling techniques have been previously employed for data reduction in databases $[1,20]$; in particular, [1] uses a sample of a large database to obtain an approximate answer. Another work, from Munro and Paterson [16], analyzed the lower bound on storage space and number of passes of a Median computation algorithm. Jain et al. [12] proposed a centralized algorithm to compute quantiles and histograms with limited storage space. Recently, Patt-Shamir [18] designed an approximate Median computation algorithm using the synopsis diffusion framework [4, 17], which uses a multipath routing algorithm to enhance robustness against communication loss. We note that none of the above algorithms were designed with security in mind, and an attacker can inject an arbitrary amount of error in the final estimate. 
Recently, a few researchers have examined security issues in aggregation algorithms. Wagner [25] addressed the problem of resilient data aggregation in the presence of malicious nodes and provided guidelines for selecting aggregation functions in a sensor network. Yang et al. [26] proposed SDAP, a secure hop-by-hop data aggregation protocol using a treebased topology to compute the Average in the presence of a few compromised nodes. SDAP divides the network into multiple groups and employs an outlier detection algorithm to detect the corrupted groups. In our extended approach, we also use a grouping technique but without any outlier detection algorithm that would otherwise require the assumption that groups have similar data distribution. Another approach for the securely computing Count and Sum, proposed by Roy et al. [22], is designed for the synopsis diffusion framework $[4,17]$.

Chan et al. [3] designed a verification algorithm by which the BS could detect if the computed aggregate was falsified. However, the authors did not propose any algorithm to compute the Median.Their verification algorithm is based on a novel method of distributing the verification responsibility onto the individual sensor nodes. An improvement on the communication complexity of the above algorithm has been recently proposed by Frikken et al. [7].

\section{ASSUMPTIONS AND PROBLEM DESCRIP. TION}

The goal of this paper is to securely compute an approximate Median of the sensor readings in a network where a few nodes might be compromised. Given a specified error bound, we return an approximate Median which is sufficiently close to the exact Median. This section describes our system model and design goals.

Network Assumptions We assume a general multihop network with a set of $\mathrm{N}$ sensor nodes and a single BS. The BS knows the IDs of the sensor nodes present in the network. The network user controls the BS, initiates the query and specifies the error bound $\epsilon$. In the rest of the paper, we consider the user and the BS as a single entity. We also consider that sensor nodes are similar to the current generation of sensor nodes (e.g., Berkeley MICA2 motes [6]) in their computational and communication capabilities and power resources, while the BS is a laptop-class device supplied with long-lasting power.

We assume that the in-network aggregation is performed over an aggregation tree which is constructed during the query broadcast, similarly as in the TAG algorithm [14]. However, our approach does not rely on a specific tree construction algorithm. The approximation error $\epsilon$ in the estimated Median $\hat{m}$ is determined by how many position $\hat{m}$ is away from the exact Median $m$ in the sorted list of all the sensed values. For ease of exposition, without loss of generality we assume that all the sensed values are distinct. Note that we could relax this assumption by defining an order on the nodes' ID that have same sensed value. Also, for the ease of exposition, we assume that there is an odd number of sensed values in total so that the Median is one element of the population.

Security Model We assume that the BS cannot be compromised. The BS uses a protocol such as $\mu$ Tesla [19] to authenticate broadcast messages. We also assume that each node $X$ shares a pairwise key, $K_{X}$ with the BS, which is used to authenticate the messages it sends to BS.

In this paper, we do not address outsider attacks - we can easily prevent unauthorized nodes from launching attacks by augmenting the aggregation framework with authentication and encryption protocols [19, 27].

We consider that the adversary can compromise a few sensor nodes (i.e., insiders) without being detected. If a node is compromised, all the information it holds will also be compromised. We use a Byzantine fault model, where the adversary can inject malicious messages into the network through the compromised nodes. We observe that a compromised node might launch multiple potential attacks against a treebased aggregation protocol, such as corrupting the underlying routing protocol, selective dropping, or a Denial of Service attack to prevent other nodes from receiving the messages from the BS. However, in this paper we address only false data injection attacks where the goal of the attacker is to cause the BS to accept a false aggregate. To achieve this goal in an in-network Median computation algorithm (e.g. $[10]$ ), a compromised node $X$ could either attempt to falsify its own sensed value, $v_{X}$, or the sub-aggregate $X$ is supposed to forward to its parent. We note that as we are computing Median, by falsifying the local value a compromised node can only deviate the final estimate by one position, i.e., the impact of the falsified local value attack is very limited. Moreover, it is impossible to detect the falsified local value attack without domain knowledge about what is an anomalous sensor reading. On the other hand, the falsified sub-aggregate attack, in which a node $X$ does not correctly aggregate the values received from $X$ 's child nodes, poses a large threat to an in-network Median computation algorithm; a compromised node $X$ forwards to its parent a corrupted aggregate which falsely summarizes $X$ 's descendants' sensed values. We observe that by launching this attack, a single compromised node, which is placed near the root on the aggregation hierarchy, can deviate the final estimate of the Median by a large amount (e.g., in [10]).

Problem Description We aim to compute an approximate Median against the falsified sub-aggregate attack. In particular, our goal is to design the following two algorithms.

- Median computation and verification algorithm: This algorithm either outputs a valid approximate Median or it detects the presence of an attack. A value, $\hat{m}$, is considered to be a valid approximate Median if it is close to the exact Median, $m$, within the bound specified by the user. In particular, if the user-specified relative error bound is $\epsilon$, the BS accepts an estimate $\hat{m}$ which satisfies the following constraint:

$$
\left|\operatorname{rank}(\hat{m})-\frac{N+1}{2}\right| \leq \epsilon N
$$

where $\operatorname{rank}(x)$ denotes the position of the value $x$ in the sorted list of all the sensed values (the population elements), and $N$ is the size of the population.

- Attack-resilient Median computation algorithm: If the above verification fails, our further aim is to compute an approximate Median in the presence of the attack.

We finally note that by launching a falsified local value attack, $w$ compromised nodes can deviate $\operatorname{rank}(\hat{m})$ in constraint (1) above by $w$ positions, which makes the error bound of the final estimate of the Median to be $(\epsilon+w / N)$. 
However, given an upper bound on $w$, the user could adjust his input $\epsilon$ to finally meet the required bound.

Notation A list of notations used in this paper is given in Table 2 .

\begin{tabular}{|c|c|}
\hline Symbol & Meaning \\
\hline$N$ & $\begin{array}{l}\text { total number of nodes } \\
\text { (or total number of sensed values) }\end{array}$ \\
\hline$S$ & sample size \\
\hline$E_{i}$ & value of $i$-th item in the sorted sample \\
\hline$K_{X}$ & $\begin{array}{l}\text { symmetric key shared between } \\
\text { node } X \text { and the BS }\end{array}$ \\
\hline$\epsilon$ & error bound for the approximate Median \\
\hline$q_{i}$ & bucket boundary in histogram \\
\hline$B_{i} \equiv\left[q_{i}, q_{i+1}\right]$ & $i$-th bucket of the histogram \\
\hline$c_{i}$ & count of $i$-th bucket \\
\hline$v_{X}$ & sensed value of node $\mathrm{X}$ \\
\hline$M A C\left(K_{X}, M\right)$ & $\begin{array}{l}\text { message authentication code of } \\
\text { message } M \text { computed using key } K_{X}\end{array}$ \\
\hline$V_{X}$ & $=\left(X, v_{X}, M A C\left(K_{X}, v_{X}\right)\right)$ \\
\hline$X \rightarrow Y$ & $\mathrm{X}$ sends a message to $\mathrm{Y}$ \\
\hline$X \rightarrow *$ & $\mathrm{X}$ broadcasts a message \\
\hline$X \Longrightarrow Y$ & $\begin{array}{l}\mathrm{X} \text { sends a message to Y } \\
\text { via multiple paths }\end{array}$ \\
\hline$a_{1} \| a_{2}$ & concatenation of string $a_{1}$ and $a_{2}$ \\
\hline$\Delta$ & $\begin{array}{l}\text { the maximum degree } \\
\text { of the aggregation tree }\end{array}$ \\
\hline$g$ & $\begin{array}{l}\text { number of groups in } \\
\text { the attack-resilient algorithms }\end{array}$ \\
\hline$w$ & number of compromised nodes \\
\hline
\end{tabular}

Table 2: Notations

\section{COMPUTING AND VERIFYING AN AP- PROXIMATE MEDIAN}

The key elements of our approach are to compute a histogram of the sensor readings and then derive an approximate Median from the histogram. We collect a sample of sensed values from the network which is used to construct the histogram bucket boundaries. Before we present our scheme, we first discuss an approach to securely compute an approximation Median whose performance will be later compared with that of our scheme. Then, we present a histogram verification algorithm and finally describe our basic scheme.

\subsection{GC Approach}

One can suggest a scheme to securely compute an approximate Median using Greenwald et al.'s Median computation algorithm [10] in conjunction with Chan et al.'s verification algorithm [3]. A brief description of these algorithms can also be found in [21]. In the first phase of $\mathrm{GC}$ approach, given the approximation error bound $\epsilon$, we can run Greenwald et al.'s algorithm to compute a quantile summary. From the quantile summary we can derive an approximate Median $\hat{m}$ which is supposed to satisfy $\epsilon$ error bound. In the next phase, we can verify the actual error present in the estimate, $\hat{m}$, which might have been falsified by an attacker in the previous phase. To verify the error, Chan et al.'s verification algorithm can be applied to count the number of nodes in the network whose value is no more than $\hat{m}$.

The communication cost per node in this approach comes from the original protocols: that is $O\left(\frac{\log ^{2} N}{\epsilon}\right)$ for Greenwald et al.'s Median computation algorithm and $O(\Delta \log N)$ for Chan et al.'s verification scheme (considering Frikken et al.'s recent improvement [7]), where $N$ is the number of nodes in the network, $\epsilon$ is the approximation error bound and $\Delta$ is the number of neighbors of a node.

\subsection{A Histogram Verification Algorithm}

We now present an algorithm for computing and verifying a histogram of sensed values, which is adapted from Chan et al.'s scheme [3] to compute and verify Sum aggregate.

Formally speaking, a histogram is a list of ordered values, $\left\{q_{0}, q_{1}, \ldots, q_{i}, \ldots\right\}$, where each pair of consecutive values $\left(q_{i}, q_{i+1}\right)$ is associated with a count $c_{i}$ which represents the number of population elements, $v_{j}$, such that $q_{i}<v_{j} \leq q_{i+1}$. We refer such an interval, $\left(q_{i}, q_{i+1}\right)$ as bucket $B_{i}$ with boundaries $q_{i}$ and $q_{i+1}$.

As noted in [3], the Sum scheme can be adapted to count the cardinality of a subset of nodes. Here, we apply Sum aggregate to count how many sensor readings belong to each histogram bucket. To do so, we require each node $X$ to contribute 1 to the count of its corresponding bucket (the bucket X's sensed value, $v_{X}$, lies within) in the histogram while we compute the total count for each bucket. Like Chan et el.'s scheme, the histogram verification scheme takes four epochs to complete: query dissemination, aggregationcommit, commitment-dissemination, and result-checking.

After an aggregation tree is constructed in the query broadcast epoch, each node $X$ 's message in the aggregation-commit epoch looks like $\left\langle\beta, c_{1}, c_{2}, \ldots, c_{b}, h\right\rangle$, where $\beta$ is the number of nodes in $X$ 's subtree, $b$ is the number of buckets in the histogram, each $c_{i}$ represents the count for the bucket $B_{i}$, i.e $\beta=\sum_{i} c_{i}$, and $h$ is an authentication field. Note that for each bucket count $c_{j}$ all of the other bucket counts together act as a complement, i.e. $c_{j}+\sum_{i \neq j} c_{i}=\beta$. A leaf node $X$ whose sensed value, $v_{X}$, lies within the bucket $B_{j}$ sets the fields in its message as follows: $\beta=1, c_{j}=1, c_{i}=0$ for all $i \neq j$, and $h=X$. If an internal node $X$ whose value $v_{X}$ lies within the bucket $B_{j}$ receives messages $u_{1}, u_{2}, \ldots, u_{t}$ from its $t$ child nodes, where $u_{k}=<\beta_{k}, c_{1}^{k}, c_{2}^{k}, \ldots, c_{b}^{k}, h_{k}>$, then $X$ 's message $\left\langle\beta, c_{1}, c_{2}, \ldots, c_{b}, h>\right.$ is generated as follows: $\beta=\sum \beta_{k}+1, c_{1}=\sum c_{1}^{k}, c_{2}=\sum c_{2}^{k}, \ldots, c_{j}=\sum c_{j}^{k}+1, \ldots$, $c_{b}=\sum c_{b}^{k}$, and $h=H\left[\beta\left\|c_{1}\right\| c_{2}\|\ldots\| c_{b}\left\|u_{1}\right\| u_{2}\|\ldots\| u_{t}\right]$, where $H$ is a hash function. The above messages along the aggregation hierarchy logically build a commitment tree which enables the authentication operation in the next phase. Once the base station receives the final commitment, it verifies the coherence of the final counts, $c_{1}, c_{2}, \ldots, c_{b}$, with the number of nodes in the network, $N$. In particular, the BS performs the following sanity check: $\sum c_{i}=N$. A simplified version of the aggregation-commit phase is illustrated in Figure 1 with an example of a small network.

Both the commitment-dissemination epoch and the resultchecking epoch are straightforward extensions of those in Chan et al.'s Sum scheme. During commitment-dissemination epoch, the final commitment is broadcast by the BS to the network. In addition, each node $X$ receives from its parent node all of the off-path values up to the root relative to $X$ 's position on the commitment tree. The aim of the commitment dissemination phase is to let each single node 


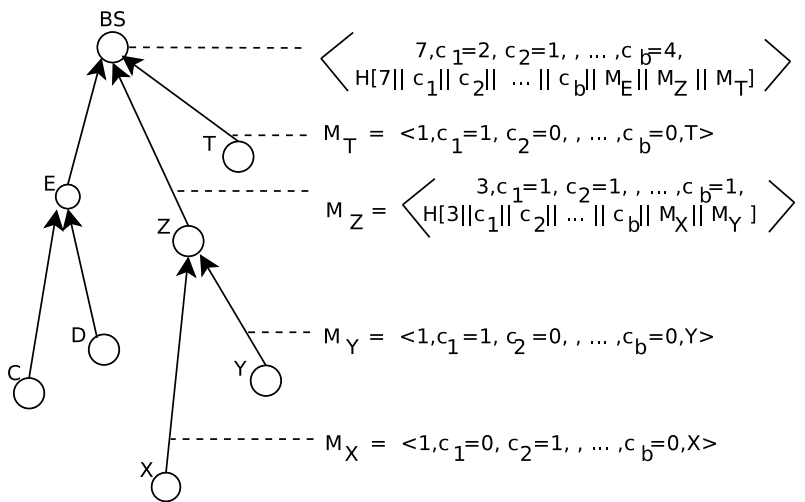

Figure 1: The aggregation-commit phase in histogram verification: In this example, $v_{X}$ lies in bucket $B_{2}, v_{Y}$ lies in bucket $B_{1}$, and $v_{Z}$ lies in the last bucket $B_{b}$.

know that its own value has been considered in the final histogram. The message containing the off-path values received by a node is bigger compared to that in the Sum scheme because each off-path value contains $b$ counts when a histogram with $b$ buckets is computed. In the result-checking epoch, the $\mathrm{BS}$ receives a compressed authentication code from all of the nodes which enables to verify if each node confirmed that its value has been considered in the final histogram.

As in Chan et al.'s Sum scheme, the main cost of this protocol is due to the dissemination of the off-path values to individual nodes. To reduce this overhead, following the recent improvement proposed by Frikken et al. [7], we use a balanced commitment tree as an overlay on the physical aggregation tree. Due to space constraint, we do not discuss the details in this paper. If a histogram with $b$ buckets is considered, each off-path message is $b$ times bigger than that in the Sum scheme, which makes the worst case node congestion in this protocol to be $O(b \Delta \log N)$.

\subsection{Our Basic Protocol}

We now describe our basic protocol to compute and verify an approximate Median. The basic protocol has two phases: sampling phase, and histogram computation and verification phase. Below we discuss these phases in detail.

While collecting a sample of population values is highly energy-efficient compared to collecting all the values, we will later show that a sample can act as a good representative of the whole population. Also, we will show that only the sample size determines the performance of our algorithm, irrespective of the size of the population.

\subsubsection{Sampling}

In this phase, the BS collects a uniform sample of the sensed values from the network. To do so, the BS broadcasts the following message:

$$
B S \rightarrow *:\langle S A M P L E, \text { seed }\rangle .
$$

The sample request coming from the BS is broadcast in a hop-by-hop fashion and the nodes arrange themselves in a ring topology; nodes at the first hop from the BS belong to the first ring and so on. A node $X$ considers the previous hop nodes as parents from which $X$ has received the query message. Note that in the sampling phase, we do not use a tree topology, which is, however, used in the histogram computation and verification phase.

We assume that there is a public hash function $F:\{I D$, seed $\} \rightarrow$ $\{0,1, \ldots, t-1\}$, where $I D$ represents the node id, seed is the nonce broadcast during the query, and $t$ is a positive integer which acts as a design parameter as discussed later. Each node, say $X$, hearing the query message applies the hash function $F(X$, seed $)$. If the resulting value is 0 , then its sensed value, $v_{X}$, is considered to be one element in the sample. In that case, $X$ computes $M A C\left(K_{X}, v_{X}\right)$ and sends the message $V_{X}=\left(X, v_{X}, M A C\left(K_{X}, v_{X}\right)\right)$ to its parents. In addition to that, if $X$ has child nodes, $X$ also forwards the sample values and corresponding MACs received from the child nodes, say $V_{Z_{1}}, \ldots, V_{Z_{c}}$. The whole message from $X$ looks as follows:

$$
X \rightarrow \operatorname{Parents}(X):\left\langle V_{X}, V_{Z_{1}}, \ldots, V_{Z_{c}}\right\rangle .
$$

When the BS receives all these messages, it verifies the corresponding MACs and outputs the list of values that are legal items of the sample. Note that the seed is used in order to have different samples in different runs. Basically, the hash function is used to uniformly divide all of the nodes among $t$ groups; the nodes belonging to the first group (i.e., output of the hash function is 0 ) are considered to constitute the sample. If the required sample size is $S$, one might set $t=N / S$. It is expected that this hash function uniformly maps $N$ elements into $t$ groups. To increase the chance that finally a sample of size no less than $S$ will be collected, we could increase the number of groups from $t$ to $k t$, and output the sample from more than $k$ groups (e.g., $k+1$ groups).

\subsubsection{Histogram computation and verification}

Once the BS obtains the sample, it sorts the items in ascending order. Then, the following steps are performed: (i) computing histogram boundaries, (ii) computing and verifying the buckets' count, and (iii) estimating the Median.

\section{i) Computing Histogram Boundaries}

We consider the number of buckets, $b$, as a parameter. In Section 5.2 we discuss how to choose this parameter. In this step, we equally divide the sample items into $b$ buckets. We denote the buckets as $B_{i}=\left[q_{i}, q_{i+1}\right], 0 \leq i \leq b-1$, where $q_{0}=-\infty, q_{i}=E_{\left\lceil\frac{S}{b}\right\rceil i}$ and $q_{b}=+\infty$, as shown in Figure 2. $E_{j}$ represents the value of $j$-th item in the sample sorted according to the value, with $j$ varying from 1 to $S$.

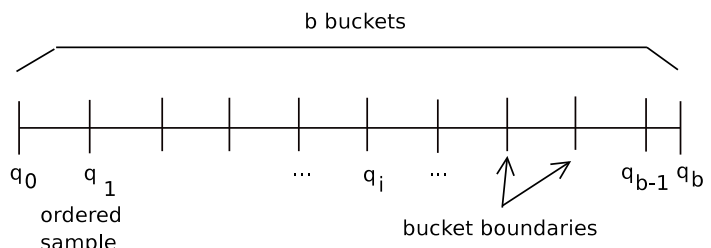

Figure 2: Computing histogram boundaries: The histogram boundaries are computed using the sample collected in the previous phase.

ii) Computing and verifying the buckets' counts

To compute the bucket counts, the BS and the sensor nodes run the histogram verification protocol described in Section 4.2. If there is no attack present in the network, 


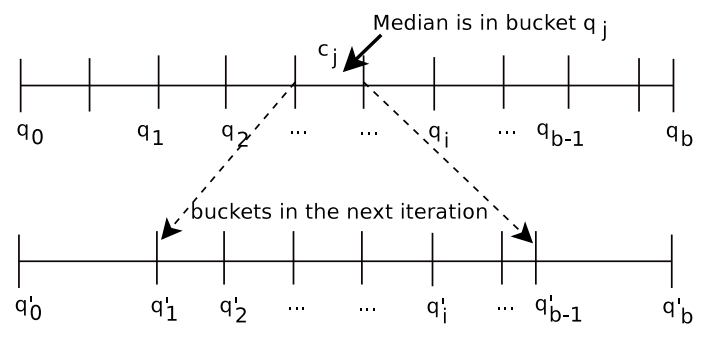

Figure 3: Splitting the bucket: If the bucket $j$, which contains the Median has more than $2 \epsilon N$ elements, the bucket is split in order to meet $\epsilon$ approximation error bound.

at the end of this step the BS knows the number of nodes that belong to each bucket in the histogram. However, an attacker node can cause this verification to fail, and in that case, the protocol terminates returning a message, "attack detected". We discuss an attack-resilient solution in Section 6 .

\section{iii) Estimating the Median}

Assuming that the verification in the previous step succeeds, we have the bucket counts $c_{0}, \ldots, c_{b-1}$ for the corresponding buckets. Our aim is now to find the bucket which contains the Median. In particular, we find $j$ such that the following three constraints are satisfied:

$$
\begin{aligned}
e_{l}+c_{0}+c_{1}+\ldots+c_{j-1} & <(N+1) / 2 \\
c_{0}+c_{1}+\ldots+c_{j} & \geq(N+1) / 2 \\
c_{j} & \leq 2 \epsilon N
\end{aligned}
$$

where $e_{l}$ is equal to 0 in the first iteration and updated as follows in other cases. We first find $j$ such that the first two in-equalities are satisfied. Then, we check if the above $j$ also satisfies in-equality (4). Note that if in-equality (4) is satisfied, then it is guaranteed that either $q_{j}$ or $q_{j+1}$ is $\epsilon N$ away from the exact Median, which is reported as our final estimate. If the in-equality (4) is not satisfied, we further split $j$-th bucket equally into $b$ sub-buckets. The new boundaries are updated as follows: $q_{0}^{\prime}=q_{0}, q_{1}^{\prime}=q_{j}, \ldots, q_{b-1}^{\prime}=q_{j+1}$, and $q_{b}^{\prime}=q_{b}$. Bucket splitting is illustrated in Figure 3. The variable $e_{l}$ is updated as $e_{l}=e_{l}+\sum_{i=0}^{j-1} c_{i}$. We iterate steps (ii) and (iii) until the in-equality (4) is satisfied. During the above iteration, if we reach a point where bucket $j$ does not contain any sample items to split further, we stop returning a message, "more sample items to be collected". We note that modifying the above inequalities any other quantiles can be computed.

\section{SECURITY AND PERFORMANCE ANAL- YSIS OF OUR BASIC PROTOCOL}

\subsection{Security Analysis}

A node $X$ which is selected in the sample sends an authentication code, $M A C\left(K_{X}, v_{X}\right)$, to the BS so that the BS can authenticate the sensed value $v_{X}$, where $K_{X}$ is the pairwise key of $X$ shared with the BS. An attacker node that is not legally selected by the hash function cannot inject a false value in the sample without being detected.

Moreover, because multipath routing scheme is used in the sampling phase, it is highly likely that we will be able to collect a sample, even if a few compromised nodes do not forward any messages. To establish the above observation, we consider a simplistic scenario. Let us assume that there are $w$ compromised nodes in total and they are randomly distributed in the network. So, the probability of a randomly selected node to be compromised is $w / N$, where $N$ is the total number of nodes. We also assume that each node has at least $\theta$ number of parents and the farthest node is $d$ hops away from the BS. We assume that unless all of the parents of a node $X$ are not compromised, $X$ 's message will reach the next hop - the probability that this happens is $(1-$ $\left.(w / N)^{\theta}\right)$. So, in the presence of the dropping attack by the compromised nodes, the probability that a sample item finally reaches the BS is at least $\left(1-(w / N)^{\theta}\right)^{d}$. As an example, with $N=1000, w=50, \theta=3$, and $d=15$, this probability is 0.998 .

Like Chan et al's scheme, our histogram computation protocol is able to detect the falsified sub-aggregate attack, i.e., the attacker cannot modify the count of any bucket in the histogram without being detected. So, given that the verification succeeds, it is guaranteed that the final estimate is an $\epsilon$-approximate Median.

\subsection{Performance Analysis}

In this section, we analyze the communication complexity of our basic protocol. In the first phase (i.e. during the sampling phase), the worst case node congestion occurs when a node (e.g. a node close to the BS) is required to forward all of the $S$ samples coming from the network. So, the maximum node congestion in the sampling phase is $O(S)$. The cost of the second phase, which computes and verifies the histogram is $O(b \Delta \log N)$, where $b$ is the number of buckets, $\Delta$ is the degree of the aggregation tree, and $N$ is number of nodes in the network. Note that our protocol iterates the second phase until the required approximation error bound is met. Our goal is to minimize the total cost of all iterations.

The second phase goes to the next iteration if the bucket $b_{j}$ in which the Median lies contains more than $2 \epsilon N$ population elements. We then further divide $j$-th bucket into $b$ subbuckets. We observe that further division is not possible if bucket $j$ no longer contains a sample item, which is bound to happen within at $\operatorname{most} \log _{b} S$ iterations. If bucket $j$ still contains more than $2 \epsilon N$ population elements, we cannot do anything further but collect more sample items.

To make an estimate of the sample size, $S$, so that we do not need to perform an extra sampling phase in most of the cases, we present the following lemma whose proof can be found in [21]

Lemma 5.1. The probability that more than $p N$ population elements lie between two consecutive items of a sorted uniform sample of size $S$ is $\phi(S, p)=(1-p)^{S-1}$, where $N$ is the population size.

As an example, from Lemma 5.1, we see that $\phi(S, 2 \epsilon)<$ $2.95 \times 10^{-5}$ for $S \geq 100$ and $\epsilon \geq 0.05$. This implies that if the user requires $\epsilon \geq 0.05$ and we use $b=10$ buckets with $S=100$, we require at most $\log _{b}(S)=2$ iterations to report the Median with probability $\left(1-2.95 \times 10^{-5}\right) \approx 1$. It is interesting to note that this result does not depend on the population size, $N$. 
Now, to measure the trade-off between the number of buckets, $b$, and the number of iterations, which together determine the total cost of the algorithm, we present the following lemma whose proof can be found in [21]

LEMmA 5.2. The probability that more than $\gamma p N(\gamma>1$, $0<p<1, \gamma p<1$ ) population elements lie between the minimum and the maximum of $p S$ consecutive sample items of a sorted sample of size $S$ is

$$
\xi(S, p, \gamma)=\sum_{i=0}^{p S}\left(\begin{array}{c}
S-1 \\
i
\end{array}\right)(\gamma p)^{i}(1-\gamma p)^{S-1-i}
$$

where $N$ is the population size.

\subsubsection{Number of buckets vs. number of iterations}

If we use $b=\frac{\gamma}{2 \epsilon}$ buckets, which is of $O\left(\frac{1}{\epsilon}\right)$, where $\gamma$ is a constant greater than 1 and $\epsilon$ is the required error bound, then each bucket contains $\frac{2 \epsilon}{\gamma} S$ sample items during the first iteration. So, the expected number of population elements in one bucket is $\frac{2 \epsilon}{\gamma} N$. In Lemma 5.2, putting $p=\frac{2 \epsilon}{\gamma}$, we can compute the probability that more than $\gamma \cdot \frac{2 \epsilon}{\gamma} \cdot N=2 \epsilon N$ population elements fall in a bucket. As Expression (5) is a decreasing function of $\gamma$, by choosing the appropriate $\gamma$, we can make the above probability close to zero. As an example, for $\gamma=2$, we observe that with sample size $S$ such that $\epsilon S \geq 5$, (i.e., each bucket contains no less than 5 sample items in the first iteration) the above probability is less than 0.02 for all $\epsilon$. That means, in this setting, our protocol ends in one iteration in $98 \%$ cases. Finally, considering the cost of the histogram verification scheme, we see that the total cost of all iterations per node, when $b=O\left(\frac{1}{\epsilon}\right)$, is $O\left(\frac{1}{\epsilon} \Delta \log N\right)$, where $\Delta$ is the degree of the aggregation tree.

On the other hand, if we use $b=O(1)$ buckets and equally divide the sample items in $b$ buckets in each iteration, then, after $\log _{b}\left(\frac{\gamma}{2 \epsilon}\right)$ iterations, each bucket will contain no more than $\frac{2 \epsilon}{\gamma} S$ sample items. So, as shown above, with the appropriate $\gamma$ chosen, it is almost certain that our algorithm will end at this point. Thus, considering the cost to compute and verify the histogram in each iteration, the total cost of all iterations, when $b=O(1)$, is $O\left(\log _{b} \frac{1}{\epsilon} \cdot b \cdot \Delta \log N\right)$, where $\Delta$ is the degree of the aggregation tree.

\section{ATTACK-RESILIENT MEDIAN COMPU- TATION}

Although our basic protocol, discussed in Section 4.3, detects falsified sub-aggregate attack, it fails to output an estimate of the Median in the presence of the attack. To address this problem, here we propose an extended approach so that we can compute an approximate Median even in the presence of a few compromised nodes.

We design the new approach based on the divide and conquer principle. We divide the network into several groups of nodes, which introduces resilience against the above attack. We run the verification algorithm individually for each group, which we call intra-group verification. Basically, we localize the attacker nodes to specific groups, i.e. we detect which groups are corrupted and which are not. Even if a few groups are corrupted, we still compute an estimate of the Median considering the valid groups. We do not assume that the groups have similar data distribution, which is the assumption exploited in other existing approaches such as SDAP [26] or RANBAR [2] .

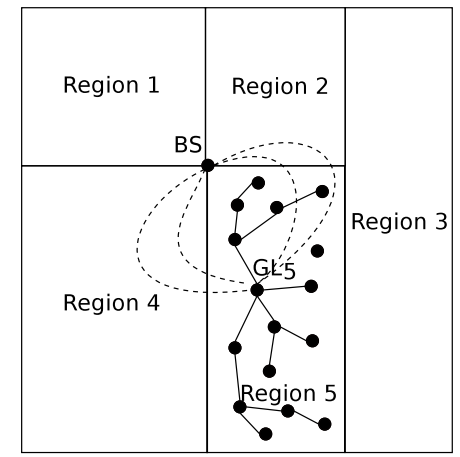

Figure 4: Geographical grouping: In each region the group leader, $G L_{i}$, sends the region aggregate to the BS by multiple paths.

We may employ different grouping techniques based on node's geographic location or node IDs. We may also use grouping technique which is based on the nodes' positions on the aggregation tree. Once the group aggregate is computed, the group leader send it directly to the BS; to avoid having any node in the middle to drop group aggregates, we use a multipath routing mechanism. Due to space constraint, only geographical grouping technique is discussed here.

Also, we may exploit the robustness property of the Median computation to determine the maximum amount of error that can be injected by a given number of corrupted nodes, even if we do not perform the intra-group verification. In [21] we estimate this error while we leave it to the network user to fix the tradeoff between the error bound and the overhead due to intra-group verification.

\subsection{Geographical Grouping}

We assume that the BS has knowledge of the location of the nodes and each node knows its own location. The network is divided into several rectangular regions, where each region is identified by a pair of geographical points. The number of regions, $g$, and the location of the regions are selected considering a few factors. As one criterion, the regions might be chosen in such a way that an equal number of nodes belong to each group - if a region has lower node density, it is likely that it will be of larger geographical size. In addition, if the BS expects that a part of the network is more likely to be under attack, it may prefer to form smaller regions in that area to better localize the attacker. Finally, The $g$ rectangular regions are specified by $g$ pairs of diametrically opposite points, $\left(x 1_{i}, y 1_{i}\right),\left(x 2_{i}, y 2_{i}\right)$, where $1 \leq i \leq g$. For each group $i$, BS also selects a node to be the group leader, $G L_{i}$. An example of this grouping is shown in Figure 4.

Once the histogram boundaries are computed using the collected sample (as in our basic protocol), the BS initiates the histogram verification procedure. The BS sends a request to the corresponding group leaders with the necessary information to identify the regions. Receiving the request, a local aggregation tree is constructed which comprises of all of the nodes in the region with $G L_{i}$ as the root. Then, the group histogram is computed locally and sent to the BS. If compromised nodes are present in a few groups, the BS will be able to identify the corrupted groups. The BS accepts 
aggregates from only those regions, which passed the verification. The BS may further split the region which contains an attacker node and run the protocol again in the subregions. Eventually, this splitting can be iterated until the attacker node is identified or the percentage of verified values satisfies the BS (e.g., when the verified groups correspond to the $95 \%$ of the nodes). Below we discuss the attack-resilient histogram computation and verification algorithm.

\subsubsection{Algorithm description}

The nodes in each region locally perform the histogram computation and verification protocol described in Section 4.2 with the group leader acting as an agent of the BS in the corresponding group. To make the group leader $G L_{i}$ an eligible agent of $B S$ for group $i$, we need a few additional communication between $G L_{i}$ and the BS. Below we focus on these additional messages skipping the detailed description of rest of the protocol, which can be found in Section 4.2. The messages exchanged between $G L_{i}$ and the BS are authenticated using their pairwise key. To improve readability, we do not show these authentication fields in the messages below.

\section{Query dissemination.}

BS initiates the query by sending to each group leader $G L_{i}$ via multiple paths the following message which contains the coordinates of the corresponding region:

$$
B S \Longrightarrow G L_{i}:\left\langle\left(x 1_{i}, y 1_{i}\right),\left(x 2_{i}, y 2_{i}\right), G L_{i}\right\rangle .
$$

In each region, the group leader, $G L_{i}$, broadcasts the received query message to its neighbor nodes, which again broadcast the same message, and so on. It is a scoped broadcast, i.e., if a node whose coordinate is outside of the corresponding region receives the message, it simply drops the message. During the query broadcast, a regional aggregation tree is formed with $G L_{i}$ as the root, similarly as in the TAG [14] algorithm. The query message also contains required $\mu T E S L A$ information (not shown above) so that each node in the region can authenticate the query.

After the query is disseminated, the nodes in each region locally perform the histogram computation and verification protocol described in Section 4.2.

\section{Aggregation-commit phase.}

After the group leader $G L_{i}$ receives the aggregated value from the nodes in group $i$, it forwards the following message to the BS:

$$
G L_{i} \Longrightarrow B S:\left\langle G L_{i}, a g g_{i}, \text { commit }_{i}\right\rangle,
$$

where $a g g_{i}$ represents the computed histogram of group $i$, and commit ${ }_{i}$ is the root of the commitment tree of region $i$.

\section{Commitment-dissemination phase.}

The BS checks if the number of nodes in the computed histogram of the group is same as the total number of nodes in that group. If yes, it sends to $G L_{i}$ the $\mu T E S L A$ authentication information, $\mu T\left(\right.$ commit $\left._{i}\right)$. So, when $G L_{i}$ broadcasts commit $_{i}$ in group $i$, each node can authenticate the message:

$$
B S \Longrightarrow G L_{i}:\left\langle G L_{i}, \mu T\left(\text { commit }_{i}\right)\right\rangle .
$$

\section{Result-checking phase.}

Each node checks if its value is incorporated in the computed histogram. If yes, node $X$ sends a MAC over an "OK" message, $M A C\left(K_{X}, O K\right)$, which gets XOR-ed with other nodes' similar messages on their way to the group leader. Once $G L_{i}$ receives the compressed OK message, say $O K_{i}$, from the nodes in its group, it forwards this message to the BS via multiple paths:

$$
G L_{i} \Longrightarrow B S:\left\langle G L_{i}, O K_{i}\right\rangle \text {. }
$$

As the BS knows which nodes belong to which group, it can verify $O K_{i}$ messages and hence can identify valid group aggregates.

\subsubsection{Security analysis}

We recall from Section that the histogram computation and verification protocol, when executed on the whole network, can detect if there is any falsified sub-aggregate attack. That means, if a malicious node $X$ fabricates the histogram of its sub-tree or if $X$ simply does not participate in the protocol, the BS can detect the attack and flags that the computed histogram is corrupted. Our intra-group verification protocol is different from the basic one only in the following aspects: (i) the histogram of the whole network is considered as the aggregate of the group histograms and each group histogram is computed and verified individually, (ii) the group leader, $G L_{i}$ exchanges a few messages with the BS, discussed in Section 6.1.1, which enable $G L_{i}$ to play the role of BS in group $i$.

The messages exchanged between $G L_{i}$ and the BS are routed via multi-paths so that they reach the destination even if an attacker node in the middle drops these messages. The communication between $G L_{i}$ and the BS is also authenticated with their pairwise key. Moreover, $G L_{i}$ receives from the BS the $\mu$ Tesla authentication information for the messages which are to be broadcast in the group, e.g., the query message and the commit $_{i}$ message. So, assuming a node $X$ knows its location, $X$ can securely determine to which group it belongs and the ID of the group leader, and $X$ can also authenticate the query and the commit ${ }_{i}$ message endorsed by the BS.

After the BS receives the group histogram from group $i$, (i.e., the $a g g_{i}$ message) the BS verifies if the number of nodes reflected in the group histogram is same as the number of nodes in the group. Also, after receiving the $O K_{i}$ message from group $i$, the BS verifies if this message correctly represents, in compressed form, the $O K$ message of all the nodes in group $i$. The above two checks enable the BS to correctly identify the corrupted groups, if any.

\subsubsection{Performance analysis}

On average, the number of nodes in one group is $N^{\prime}=$ $\frac{N}{g}$, where the network is divided into $g$ groups. So, the worst case node congestion inside one group for running the histogram verification algorithm is $O\left(b \cdot \Delta \cdot \log N^{\prime}\right)$, where $b$ is the number of buckets in the histogram and $\Delta$ is the number of neighbors of a node on the aggregation tree. Considering the analysis given in Section 5.2.1, with $b=O\left(\frac{1}{\epsilon}\right)$, the worst case communication overhead per node is $O\left(\frac{1}{\epsilon} \cdot \Delta \cdot \log N^{\prime}\right)$. In addition, a node needs to forward the messages exchanged between the group leaders and the BS, which is of $O(g)$ communication overhead in the worst case.

\section{SIMULATION RESULTS}

In this section, we report on a simulation study that examined the performance of our basic protocol discussed in 
Section 4. Recall that, in the first phase, we collect a sample of sensed values from the network, and the performance of the rest of the protocol depends on the quality of this sample. The goal of the simulation experiments reported below is to study the impact of the sample on the overall performance of the Median computation protocol. In particular, we verify the results we obtained via analysis, in Section 5.2, about the inter-relationship among parameters, such as error bound $\epsilon$, sample size $S$, and the number of buckets $b$ in the histogram.

Through simulation we do not evaluate the overhead of in-network communications in our protocol. The analytical results on the communication overhead of the sampling phase and the histogram computation and verification phase are discussed in Section 5.2.

\subsection{Simulation Environment}

In our basic setup, the network size is 1,000 nodes. We also vary the network size to show that it does not have a significant impact on our sampling-based approach. In our simulation, the typical value we take for the $\epsilon$ error bound varies from $5 \%$ to $15 \%$. Each node has one sensed value, while our goal is to compute an approximate Median. We use the method of independent replications as our simulation methodology. Each simulation experiment was repeated no less than 1000 times with different seeds.

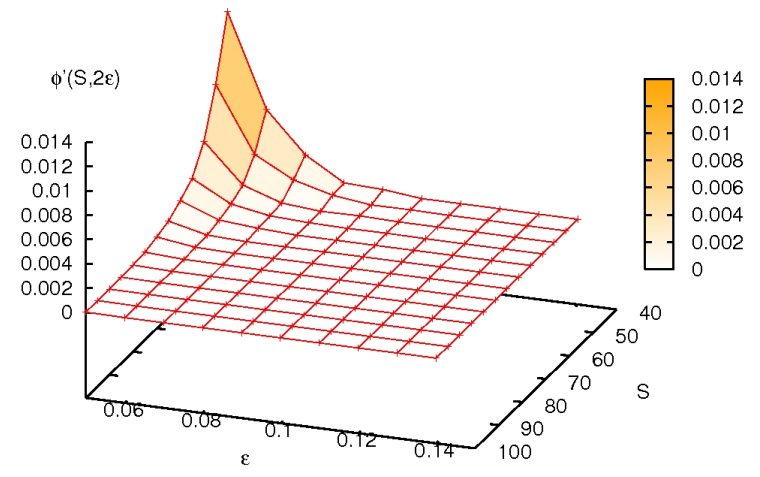

Figure 5: Computing the chance that we need to collect more sample items: Given an $\epsilon$, we choose a sample size so that the probability that we need to redo the sampling is close to zero.

\subsection{Results and Discussion}

Here, we discuss the results obtained in our simulations. We observe that $95 \%$ confidence interval of all the quantities on the following plots are within $5 \%$ of the reported value.

What is the chance that one sampling phase is not enough? In Lemma 5.1, we analytically computes this probability which we evaluate via simulation here. For each pair $(S, \epsilon)$, we collect a sample of size $S$ and we compute the number of time, $\tau$ there are more than $2 \epsilon N$ elements between the two consecutive sample items containing the Median. The total number of runs performed is 1,000,000. The resulting $\phi^{\prime}(S, 2 \epsilon)$, which is the observed approximation

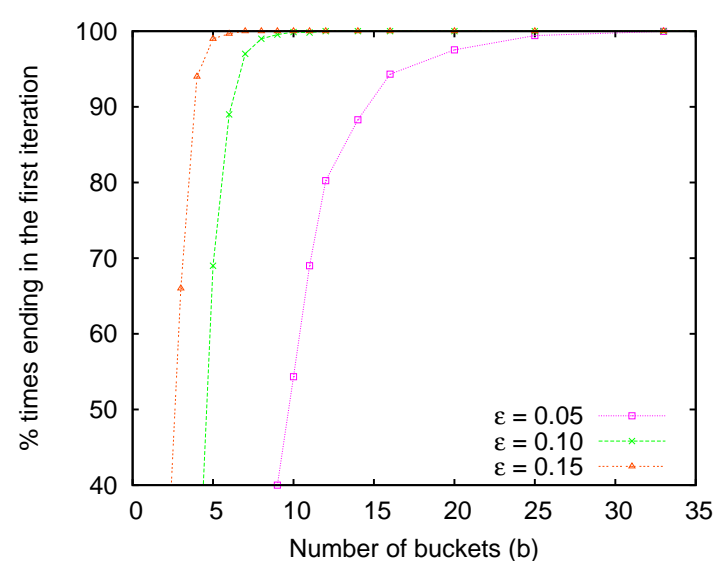

(a) $\%$ Times ending in one iteration

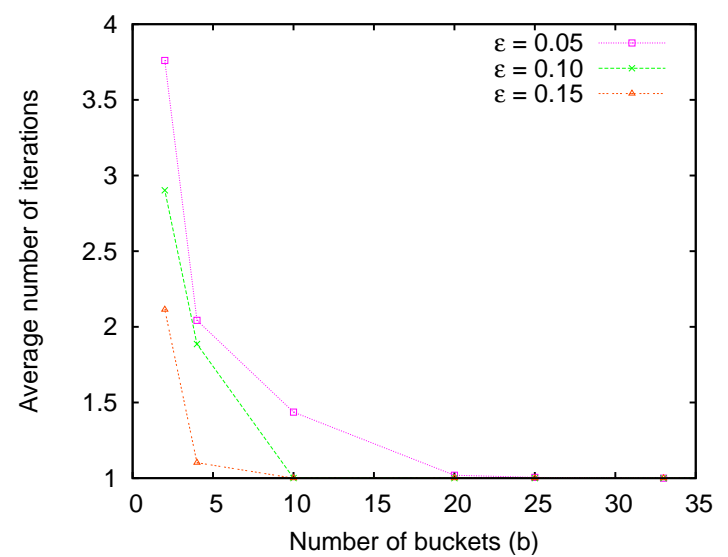

(b) Average number of iterations

Figure 6: The number of iterations vs. the number of buckets: if the number of buckets is $O\left(\frac{1}{\epsilon}\right)$, it is highly likely that our algorithm ends in one iteration.

of $\phi(S, 2 \epsilon)$, is plotted in Figure 5. It is worth noticing that the value of $\phi^{\prime}(S, 2 \epsilon)$ is less than $4 \times 10^{-5}$ for $\epsilon>0.05$ when the sample size $S$ is more than 95 . In fact, as expected, for a given $\epsilon$, an increase of the value of $S$ decreases $\phi^{\prime}(S, 2 \epsilon)$. Finally, we verify that $\phi^{\prime}(S, 2 \epsilon)$ does not change significantly (not shown in the figure) even if the population size, $N$, is bigger.

Number of buckets vs. Number of iterations. In Section 5.2, we analyzed the dependence of the number of iterations of our algorithm on the number of buckets chosen, which we validate here via simulations. First, we estimate the number of buckets required to end our protocol in one iteration in most cases. Figure 6 (a) illustrates the $\%$ of cases our protocol ends in the first iteration. The figure confirms our analysis that, for considering $\gamma=2$, if we use more than $\frac{1}{\epsilon}$ buckets (i.e., 20, 10, 7 buckets for $\epsilon=0.05,0.10,0.15$, respectively), it is highly likely that we need just one iteration. Finally, Figure 6(b) shows the average number of iterations required using different number of buckets, where $\epsilon=0.05$ and $S=100$. This validates our analysis that the average number of iterations is $O\left(\log _{b}\left(\frac{1}{\epsilon}\right)\right)$ when $b$ buckets are used. 


\section{CONCLUSION}

While researchers already addressed the problem of securely computing aggregates such as Sum, Count, and Average, to the best of our knowledge, there is no prior work on secure computation of the Median. However, it is widely considered that the Median is an important aggregate. In this paper, we proposed a protocol to compute an approximate Median and verify if it is falsified by an attack. Once the protocol is executed, the base station either possesses a valid approximate Median or it has detected an attack. Further, we proposed an attack-resilient algorithm to compute the Median even in the presence of a few compromised nodes. The evaluation via both analysis and simulation shows that our approach is efficient and secure.

\section{REFERENCES}

[1] D. Barbará, W. DuMouchel, C. Faloutsos, P. J. Haas, J. M. Hellerstein, Y. E. Ioannidis, H. V. Jagadish, T. Johnson, R. T. Ng, V. Poosala, K. A. Ross, and K. C. Sevcik. The new jersey data reduction report. IEEE Data Eng. Bull., 20(4):3-45, 1997.

[2] L. Buttyán, P. Schaffer, and I. Vajda. RANBAR: RANSAC-based resilient aggregation in sensor networks. In $S A S N$ '06, pages 83-90, 2006.

[3] H. Chan, A. Perrig, and D. Song. Secure hierarchical in-network aggregation in sensor networks. In $C C S$ '06: Proceedings of the 13th ACM Conference on Computer and communications security, pages 278-287, 2006

[4] J. Considine, F. Li, G. Kollios, and J. Byers. Approximate aggregation techniques for sensor databases. In ICDE '04: Proceedings of the 20th International Conference on Data Engineering, pages 449-460, 2004.

[5] G. Cormode and S. Muthukrishnan. An improved data stream summary: The count-min sketch and its applications. In LATIN '04: Proceedings of Latin American Theoretical Informatics, pages 29-38, 2004.

[6] Crossbow Technology Inc. http://www.xbow.com, 2008.

[7] K. Frikken. An efficient integrity-preserving scheme for hierarchical sensor aggregation. In WiSec '08: Proceedings of the First ACM Conference on Wireless Network Security, pages 68-76, 2008.

[8] W. F. Fung, D. Sun, and J. Gehrke. Cougar: the network is the database. In SIGMOD '02: Proceedings of the 2002 ACM SIGMOD international conference on Management of data, pages 621-621, 2002.

[9] M. Greenwald and S. Khanna. Space-efficient online computation of quantile summaries. In SIGMOD '01: Proceedings of the 2001 ACM SIGMOD international conference on Management of data, pages 58-66, 2001

[10] M. B. Greenwald and S. Khanna. Power-conserving computation of order-statistics over sensor networks. In PODS '04: Proceedings of the twenty-third ACM SIGMOD-SIGACT-SIGART symposium on Principles of database systems, pages 275-285, 2004.

[11] Habitat Monitoring on Great Duck Island. http://www.greatduckisland.net/.

[12] R. Jain and I. Chlamtac. The $P^{2}$ algorithm for dynamic calculation of quantiles and histograms without storing observations. Communications of the
ACM, 28(10):1076-1085, October 1985.

[13] James Reserve Microclimate and Video Remote Sensing. http://www.cens.ucla.edu.

[14] S. Madden, M. J. Franklin, J. M. Hellerstein, and W. Hong. TAG: a tiny aggregation service for ad-hoc sensor networks. In OSDI '02: Proceedings of the 5th symposium on Operating systems design and implementation, pages 131-146, 2002.

[15] G. S. Manku, S. Rajagopalan, and B. G. Lindsay. Approximate medians and other quantiles in one pass and with limited memory. SIGMOD Record, 27(2):426-435, 1998.

[16] J. I. Munro and M. S. Paterson. Selection and sorting with limited storage. Theoretical Computer Science, (12):315-323, 1980.

[17] S. Nath, P. B. Gibbons, S. Seshan, and Z. R. Anderson. Synopsis diffusion for robust aggregation in sensor networks. In SenSys '04: Proceedings of the 2nd international conference on embedded networked sensor systems, pages 250-262, 2004.

[18] B. Patt-Shamir. A note on efficient aggregate queries in sensor networks. Theoretical Computer Science, 370(1-3):254-264, 2007.

[19] A. Perrig, R. Szewczyk, V. Wen, D. Culler, and J. D. Tygar. SPINS: Security protocols for sensor networks. Wireless Networks, 8(5):521-534, Sept. 2002.

[20] V. Poosala, P. J. Haas, Y. E. Ioannidis, and E. J. Shekita. Improved histograms for selectivity estimation of range predicates. SIGMOD Record, 25(2):294-305, 1996

[21] S. Roy, M. Conti, S. Setia, and S. Jajodia. Attack-resilient hierarchical data aggregation in sensor networks. Technical report, Center for Secure Information Systems - George Mason University (http://mason.gmu.edu/ sroy1/tr-median.pdf), March 2008.

[22] S. Roy, S. Setia, and S. Jajodia. Attack-resilient hierarchical data aggregation in sensor networks. In SASN '06: Proceedings of the fourth ACM workshop on Security of ad hoc and sensor networks, pages 71-82, 2006.

[23] N. Shrivastava, C. Buragohain, D. Agrawal, and S. Suri. Medians and beyond: new aggregation techniques for sensor networks. In SenSys '04: Proceedings of the 2nd international conference on embedded networked sensor systems, pages 239-249, 2004.

[24] The Firebug Project. http://firebug.sourceforge.net.

[25] D. Wagner. Resilient aggregation in sensor networks. In SASN '04: Proceedings of the 2nd ACM workshop on Security of ad hoc and sensor networks, pages 78-87, 2004.

[26] Y. Yang, X. Wang, S. Zhu, and G. Cao. SDAP: a secure hop-by-hop data aggregation protocol for sensor networks. In MobiHoc '06: Proceedings of the 7th ACM international symposium on Mobile ad hoc networking and computing, pages 356-367, 2006.

[27] S. Zhu, S. Setia, and S. Jajodia. Leap: efficient security mechanisms for large-scale distributed sensor networks. In CCS '03: Proceedings of the 10th ACM conference on Computer and communications security, pages $62-72,2003$ 